\title{
A Non-invasive Blood Glucose Monitoring System Based on Smartphone PPG Signal Processing and Machine Learning
}

\begin{abstract}
Blood glucose needs to be monitored on a regular basis to prevent diabetes from consuming the health of hyperglycemic patients. Currently in clinic, it is measured using an invasive technique which is uncomfortable and has risky of infection. To facilitate daily care at home, we propose an intelligent, non-invasive blood glucose monitoring system which can differentiate a user's blood glucose level into normal, borderline and warning based on smartphone PPG signals. The main implementation processes of the proposed system include: (1) a novel algorithm for acquiring photoplethysmography (PPG) signals using only smartphone camera videos; (2) a fitting-based sliding window (FSW) algorithm to remove varying degrees of baseline drifts and segment the signal into single periods; (3) extracting characteristic features from the Gaussian functions by comparing PPG signals at different blood glucose levels; (4) categorizing the valid samples into three glucose levels by applying machine learning algorithms. Our proposed system was evaluated on a data set of 80 subjects. Experimental results demonstrate that the system can separate valid signals from invalid ones at an accuracy of $97.54 \%$ and the overall accuracy of estimating the blood glucose levels reaches $81.49 \%$. The proposed system provides a reference for the introduction of non-invasive blood glucose technology into daily or clinical applications. This research also indicates that smartphone-based PPG signals have great potentiality to assess individual's blood glucose level.
\end{abstract}

Index Terms-Non-invasive Blood Glucose Monitoring, Smartphone PPG Signal, Gaussian Fitting, Healthcare based on Machine Learning, Daily Care.

\section{INTRODUCTION}

D IABETES is a lifelong metabolic disease characterized by chronic hyperglycemia due to multiple causes such as impaired insulin secretion and impaired biological effects [1]. Currently, there is no effective treatment of diabetes, but regular monitoring of blood glucose level can reduce or delay the incidence of complications [2]. Self-monitoring is considered as one of the most direct and feasible options for controlling diabetes [3].

The mature detection technique is to use a blood glucose analyzer to measure the blood of a patient obtained by piercing a finger. This method not only brings pain and economic burden to patients due to frequent blood collection, but also does not allow real-time monitoring [4], [5]. Non-invasive blood glucose technology can overcome the above shortcomings and has become a hot issue of smart healthcare research [6]. However, non-invasive blood glucose monitoring system that can be used at home for self-monitoring is still in the early stage of development.

Photoplethysmography (PPG) technique has been applied to measure blood volume changes in certain parts of the body [7]. A PPG device usually consists of a light source for illuminating the tissue, and a detector for sensing the reflected light, respectively. The amount of light absorbed varies periodically according to fluctuations of blood volume in the circulatory system, causing the PPG signals to contain information related to breathing, circulatory system, blood flow and heartbeat [8]. Nabeel [9] realized non-invasive blood pressure measurement, and Motin [10] performed effective heart rate and respiratory rate monitoring. Both of the two works are accomplished by analyzing specific components in the PPG signal. Processing PPG signal can reveal new information on the body's hemodynamic characteristics and certain blood parameters, which are of great significance for personal health mornitoring [11].

At present, most of the human physiological measurements using photoelectric information is rely on professional acquisition equipments. In [12], PPG signals from normal and hyperglycemic patients were obtained using a Glutrac connected to cloud processing system. Although this method can obtain high quality data, it has certain cost limitations and difficulties to achieve universalization. Therefore, we aim to obtain PPG signals through the smartphone camera by analyzing the variation of light intensity from the change of blood volume in the finger.

A major breakthrough has been made in the combination of artificial intelligence and medical fields which is widely applied for clinical decision support and medical image processing. Our proposed system utilizes machine learning algorithms to estimate blood glucose levels via PPG signals retrieved from smartphone. Our main contributions include:

- A few methods for PPG signal retrieval have been developed in literature, however most of them rely on professional equipments. We propose a novel algorithm to extract PPG signals from only smartphone video. Experimental results indicate that our algorithm is effective for PPG signals which are extremely weak and susceptible to noise interference. Consequently our method fits well with home self-monitoring due to its portability and ease of use.

- Traditional approaches usually removed baseline drift through high-pass filters. Considering the fact that signal components are complex and there exist varying degrees of baseline drift, we develop a fitting-based sliding (FSW) algorithm to remove baseline drift meanwhile dividing the signals into single periods. Therefore, the original characteristics of the PPG signals are retained to a great extent, meanwhile distortion introduced by filtering is avoided.

- We propose a feature extraction method based on Gaus- 
sian fitting to find out the correspondence between the hyperglycemia and the normal human PPG signals in both time domain and frequency domain by analyzing the parameters of the positive Gaussian function. Experimental results suggest that features extracted by this method can efficaciously divide blood glucose into three levels (normal, borderline and warning).

The rest of the paper is organized as follows. Section II presents the related work. Section III describes the proposed methodology in detail. Results obtained by experiment are explained in section IV and section $\mathrm{V}$ concludes the paper.

\section{RELATED WORK}

Non-invasively tracking blood glucose levels is now possible due to various breakthroughs in optics and electrochemistry. We focus on the work related to measuring blood glucose based on PPG signals and summarize the representative researches as follows.

In [1], PPG reflecting the heart cycle was acquired according to different optical intensity through the self-made embedded finger clip, composed of LED and photodiode. According to the authors' research, the transmittance of light is inversely proportional to the blood glucose concentration. The acquired signal was converted into the digital signal by combining highpass filter, amplifier and fourth-order low-pass filter to eliminate the high frequency noise. The motion artifacts were then processed using an adaptive adaline neural network filter. The invasive blood glucose meter was used to label the subject's actual blood glucose level, which is then combined with the filtered PPG signal to predict blood glucose levels using an artificial neural network integrated into a field programmable gate array (FPGA). The data were from 50 individuals with different blood glucose levels, and the estimated accuracy was 95.38\%. Light conditions and adipose tissue can affect system performance. The acquisition of the signal was simple and portable, but the processing was complicated, and it could not offer the advantage of real-time monitoring.

Hina [13] presented a non-invasive glucose monitoring prototype system composing of three main modules. The first module, consisting of a clip and a LED, was implemented on a printed circuit board (PCB) to obtain PPG signals. The second module performed signal preprocessing whereafter 10 features were extracted on the FPGA. The third module used a machine learning index GPR model to measure blood glucose levels. Data were collected from 200 volunteers including 4 diabetic patients. The mean absolute relative difference (mARD) in predicted blood glucose levels was $8.97 \%$. The acquired signal was also transmitted to a computer for further processing, which is time consuming.

Monte-Moreno [14] proposed the use of respiratory frequency, heart rate, vascular compliance, blood viscosity and other physiological parameters to analyze the PPG waveform and then estimate blood glucose. After acquiring the PPG by the finger clip, denoising was performed to remove motion artifacts for feature extraction. Finally, among SVM, random forest, linear regression and neural network classifiers, random forest performed better than other classifiers. Using Clark

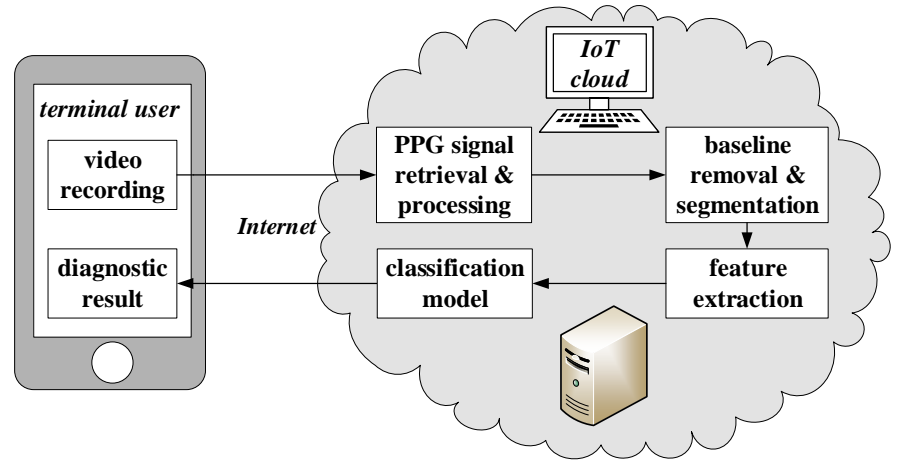

Fig. 1. Smartphone PPG based non-invasive blood glucose monitoring system.

Grid Analysis (CGA), $87.7 \%$ of the points fell into Zone A. Tsai [12] used a wearable health device called Glutrac to obtain PPG signals from fingers and wrists, and then sent the acquired signals to NoSQL databases via Bluetooth 5.0 for cloud storage and calculation. After signal denoising, 30 features were extracted from the time domain and derivatives. In the experiment, multiple decision trees were used for data learning. Nine patients with type 2 diabetes participated in the trial and invasive blood glucose values were obtained by finger puncture using Accuc-chek. The global accuracy of the model reached $80 \%$, however the accuracy varied greatly between different individuals. Rachim [15] collected PPG signals under different optical wavelengths by wearable biosensors. Digital wavelet transform (DWT) and wavelet decomposition (WD) were used to remove the baseline wander of the original PPG signals. After executing the local maxima algorithm for signal peak detection, 24 features were extracted from each periodic PPG signal. The model was based on a linear partial least squares (PLS) multivariate calibration and was evaluated by 10 -fold cross validation. A total of 12 subjects participated in the experiment. The standard error of prediction (SEP) and the correlation coefficient $\left(R_{p}\right)$ between the estimated and the reference blood glucose were $6.16 \mathrm{mg} / \mathrm{dL}$ and 0.86 , respectively. The above three methods require individual-based calibration and can not satisfy multi-user scenarios.

To sum up, there is up to now no non-invasive blood glucose monitoring system based on PPG signals from smartphone, which has the advantages of portability, time efficiency and no calibration requirement.

\section{PROPOSED SYSTEM}

Users can record video and acquire blood glucose level through a smartphone. Signal retrieval and preprocessing are performed in the cloud, and the final result is obtained after feature extraction and classification. Fig. 1 shows the proposed system functional flowchart. Blood glucose scale is divided into 3 groups ranging from $3.9 \mathrm{mmol} / \mathrm{L}$ to $11 \mathrm{mmol} / \mathrm{L}$, and the corresponding levels are also listed in the table I.

\section{A. Smartphone Video Recording}

A 60-second video of the left index finger was recorded using a 30-frame-rate (sampling rate $30 \mathrm{~Hz}$ ) iPhone 6s Plus 
TABLE I

BLOOD GLUCOSE GROUPS

\begin{tabular}{ccc}
\hline Groups & Blood Glucose Ranges $(\mathrm{mmol} / \mathrm{L})$ & level \\
\hline G1 & $3.9-6.1$ & Normal \\
G2 & $6.2-7.8$ & Borderline \\
G3 & $7.9-11$ & Warning \\
\hline
\end{tabular}

TABLE II

CONDITIONS FOR EVALUATING Video USABILITY.

\begin{tabular}{|c|}
\hline $\operatorname{Ave}(\mathrm{R}) \geq 240$ \\
\hline$\sigma \mathrm{R} \leq 20$ \\
\hline $\operatorname{Ave}(\mathrm{G})<1$ \\
\hline $\operatorname{Ave}(\mathrm{B})<75$ \\
\hline
\end{tabular}

camera. Fingertip must cover both the flash and the camera simultaneously. Light and dark switched obviously in the video as the finger's blood volume varies, whereafter an invasive ACCU-CHEK Advantage blood glucose meter complying with the international standard ISO15197 (2013) was adopted to detect the actual blood glucose value for labeling (3 groups). These two procedures were performed consecutively but separately (the interval was less than one minute), so that the blood glucose level did not fluctuate in such a short time. The video was then transferred to a computer for further processing by MATLAB.

Appropriate video recording is subject to the position of fingertip on the camera, the pressure of fingertip and the lighting conditions [16]. Therefore, a usability evaluation scheme for the smartphone video is proposed to monitor the saturation of each color channel in the frame. Chromatic parameters including average value of the red $\operatorname{Ave}(R)$, green $\operatorname{Ave}(G)$ and blue Ave(B) channels, as well as the corresponding standard deviation of the red channel $\sigma \mathrm{R}$, are evaluated frame by frame. We implemented a statistical analysis of each chrominance parameter to provide a suitable threshold. A total of 3000 video frames from the first 5 seconds of 20 subjects under correct operations were used to calculate and select the critical value of the distribution range of each parameter as the threshold.

Table II summarizes the conditions for proper video recording. If the chromatic parameters in a certain frame do not meet the above conditions, it means that video has not been properly recorded. It should be noted that the evaluation of the video usability is only for preliminary screening to ensure a user's correct operation so that PPG signals could be extracted. The removal of the unavoidable baseline drift is described in subsection III.C.

Table III presents four frames among which the first two frames are recognized to be operationally correct and can be accepted for further analysis. Chrominance parameters of the last two frames do not meet the requirements specified, hence are discarded.

\section{B. PPG Signal Retrieval}

The absorption of light by blood is associated with the change of finger blood volume, which is reflected in the video. Accordingly, the pixel intensity of the same area in adjacent frames is different. We calculate a continuous PPG signal
TABLE III

EXAMPLE OF EVALUATING VIDEO USABILITY.

\begin{tabular}{|c|c|c|}
\hline Frame & $\begin{array}{l}\text { Chromatic } \\
\text { parameters }\end{array}$ & Note \\
\hline & $\begin{array}{c}\operatorname{Ave}(\mathrm{R})=244.11 \\
\sigma(\mathrm{R})=15.76 \\
\operatorname{Ave}(\mathrm{G})=0.23 \\
\operatorname{Ave}(\mathrm{B})=32.37\end{array}$ & Acceptable \\
\hline & $\begin{array}{c}\operatorname{Ave}(\mathrm{R})=245.37 \\
\sigma(\mathrm{R})=19.17 \\
\operatorname{Ave}(\mathrm{G})=0.15 \\
\operatorname{Ave}(\mathrm{B})=70.33\end{array}$ & Acceptable \\
\hline & $\begin{array}{c}\operatorname{Ave}(\mathrm{R})=254.22 \\
\sigma(\mathrm{R})=1.33 \\
\operatorname{Ave}(\mathrm{G})=22.20 \\
\operatorname{Ave}(\mathrm{B})=127.87\end{array}$ & $\begin{array}{c}\text { Not } \\
\text { Acceptable }\end{array}$ \\
\hline & $\begin{array}{c}\operatorname{Ave}(\mathrm{R})=123.03 \\
\sigma(\mathrm{R})=82.28 \\
\operatorname{Ave}(\mathrm{G})=113.16 \\
\operatorname{Ave}(\mathrm{B})=99.93\end{array}$ & $\begin{array}{c}\text { Not } \\
\text { Acceptable }\end{array}$ \\
\hline
\end{tabular}

based on the overall changes of pixel intensity in each video frame. The red, green and blue channels are separated from a single frame of the correctly recorded video, as shown in Fig. 2(a) and 2(b). A threshold is set for each frame using Eq. (1), and the PPG value of frame $i$ is calculated by Eq. (2) which is the sum of the pixels with intensity greater than a specified threshold. PPG signal is then obtained by plotting the calculated sums of each frame pixel.

$$
\begin{gathered}
\text { Threshold }=1.01 \times\left(\text { intensity }_{\max }-\text { intensity }_{\min }\right) \\
P P G_{\text {signal }}[i]=\sum_{1}^{\text {total pixels }} \text { inensity }^{\text {tot }}>\text { Threshold }
\end{gathered}
$$

The selection of threshold is determined by a large number of attempts. When threshold gets lower and the output becomes smoother, signal eventually turns into a horizontal line. If threshold keeps getting higher, the signal will gradually reach some peaks, which will affect the signal quality. Taking full consideration of the waveform accuracy and without loss of generality, threshold in our proposed system is defined as 1.01 times of the pixel intensity range.

To determine the channel that can generate PPG signals after a simple calculation, the signals are extracted from all three channels (red, green, blue) as shown in Fig. 2(c). It is evident that PPG signals obtained from the red channel are the clearest and do not require further processing to reflect the periodic variation of blood volume during cardiac cycles. While green and blue channels are difficult to extract useful information without other complicated processing.

\section{Raw PPG Signal Preprocessing}

When the light falls on the skin, it will be detected by the photodiode in two modes: transmission mode where the 
(a)

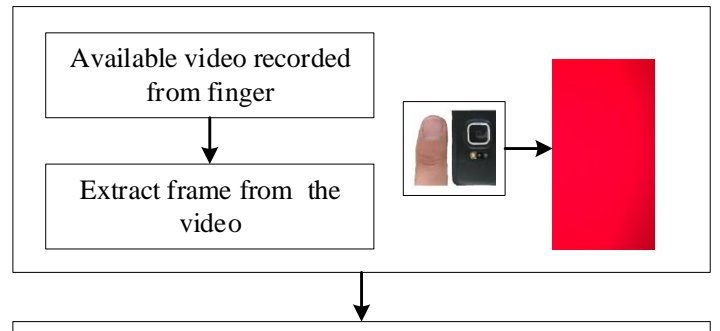

(b)

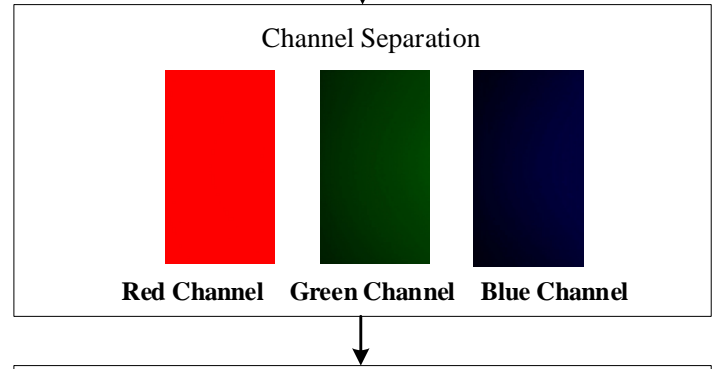

(c)

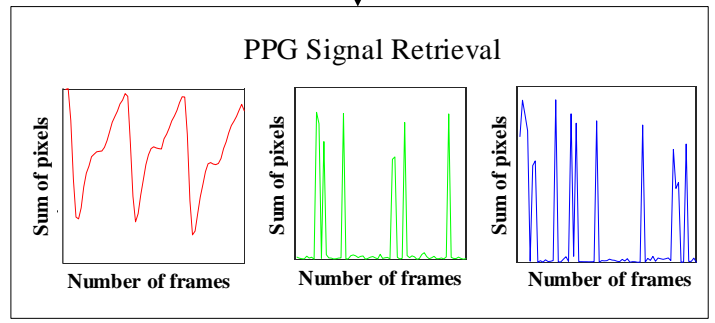

Fig. 2. PPG signal retrieval methodology. (a) video recording using smartphone camera and frame extraction from the video, (b) red, green and blue components of a frame, (c) PPG signal retrieval from the red, green and blue components, respectively.

light source and the photo-detector are on the opposite side of each other, and reflection mode where the light source and the photo-detector are on the same side. In our scenario, light from the smartphone's flash is captured by the camera on the same side due to reflections from the tissue, bones and blood vessels of the finger [17], [18]. Therefore, the original PPG signals retrieved from the recorded video are reversed and need to be inverted to recover to normal case as shown in Fig. 3(a) and 3(b), respectively. Reflective mode PPG signals are susceptible to noise such as ambient light, temperature, power frequency interference so that the inverted signals are denoised by a sixorder Butterworth filter to remove the frequency components higher than $16 \mathrm{~Hz}$ after Fourier analysis, as shown in Fig. 3(c).

During the video capture, the smartphone camera is simply attached to the fingertip, easily introducing motion artifacts to the extracted PPG signals. Although this does not lead to significant baseline drifts, the inherent waveform characteristics of the raw single-period signals will be distorted. Removing low-frequency components to get rid of the baseline drifts will result in the loss of some useful information in the waveform. Therefore, the details should be better preserved to ensure that the pathological features of the signal are not lost.

Sliding window algorithm is widely adopted for trending and rhythm analysis of continuous dynamic physiological signals to explore pathological information. In [19], feature extraction and heart rate calculation of PPG signals were performed using sliding window algorithm. In [20], sliding window was used to find peaks and valleys. However, due (a)

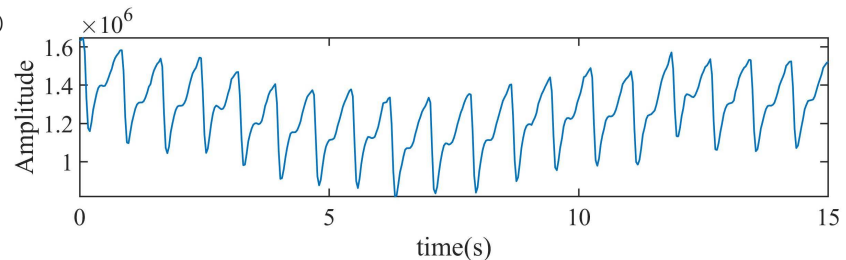

(b)

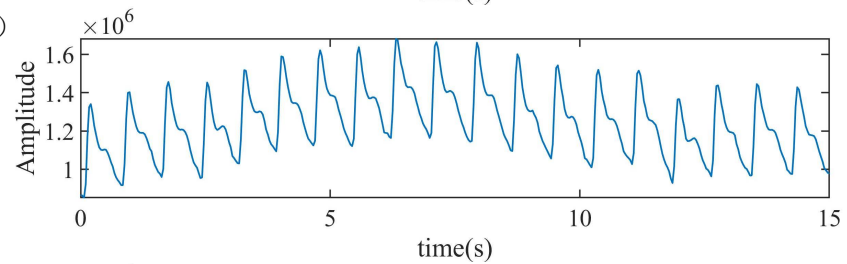

(c)

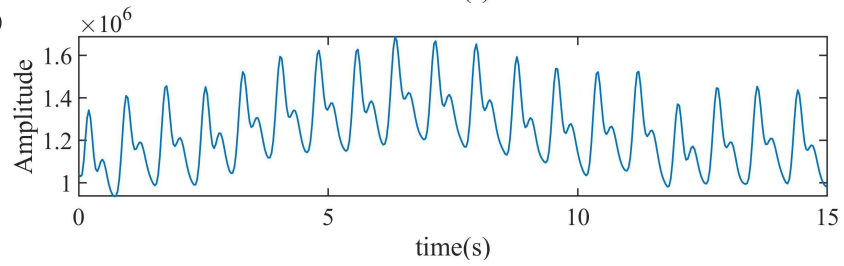

Fig. 3. Signal preprocessing stage. (a) Raw smartphone PPG signal, (b) Inverted smartphone PPG signal, (c) Filtered smartphone PPG signal.

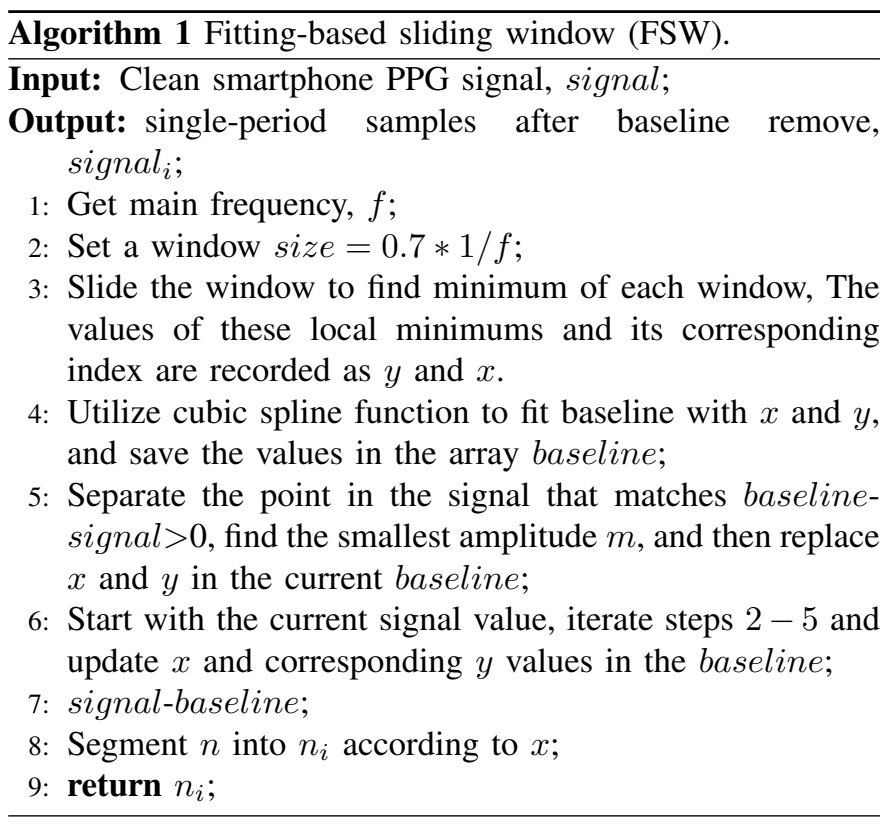

to the existence of baseline drift, the valleys were not well recognized. Once the valley was identified incorrectly, the probability of the next valley being wrong became higher. Therefore the valleys should be identified as accurately as possible. Consequently, we propose FSW algorithm to remove baseline drift, after that signal is brought back to its normal base (x-axis) and segmented into single periods. The valleys of the PPG signal are accurately recognized using a sliding window and through an automatic error correction mechanism. The cubic spline is utilized to fit the baseline of the signal with these valleys.

The pseudocode in Algorithm 1 provides the entire process of the FSW algorithm. For the purpose of periodic segmentation, correctly localizing all the valleys of the continuous PPG 


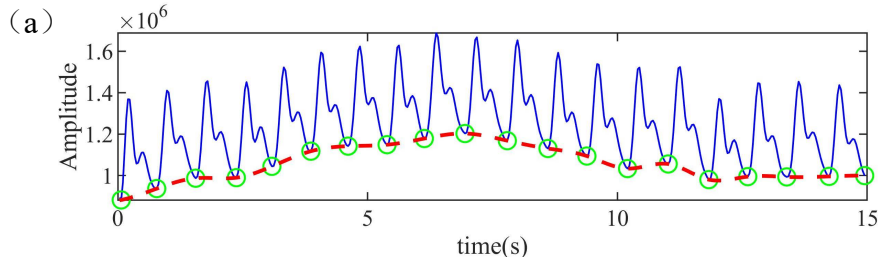

(b)

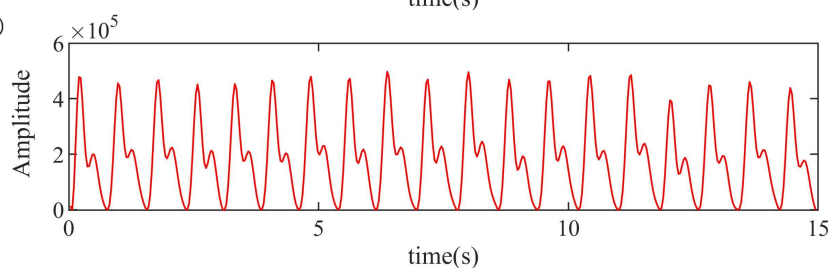

Fig. 4. Process of the FSW algorithm. (a) Baseline fitted with cubic spline interpolation: green circle is the valleys and red dotted line is the baseline, (b) PPG signal baseline removal.

signals is a key prerequisite. Through extensive experiments, we set the window size to be $7 / 10$ period, among all the possibilities, to realize good performance in terms of accuracy and real time response. The period length is obtained by analyzing the frequency of the periodic signal. One advantage of FSW algorithm lies in its automatic correction of those valleys mistakenly identified. Once the baseline is noticed to be greater than the signal, the minimum value of the segmented signal will be adopted as the new valley from which the window will be re-established to update the subsequent valley value. In this case the loop body ends when no baseline is greater than the signal.

Fig. 4(a) indicates that FSW algorithm performes well in identifying valleys of the continuous PPG signals. No obvious distortion is introduced to the signals after baseline drift removal, as shown in Fig. 4(b). The signal is then divided into single periods. The waveform of single-period PPG signals might vary slightly for different people, but they are similar in terms of characteristics. After FSW algorithm implementation, certain parts of the damaged single-period signal might be incorrectly identified. As a result, a segmented single-period signal may comprise the falling branch of the previous signal or the rising branch of the following signal, which is usually wider than a normal signal and apparently invalid as shown in Fig. 5(b).

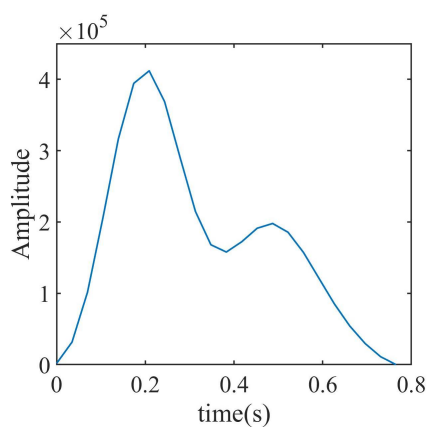

(a)

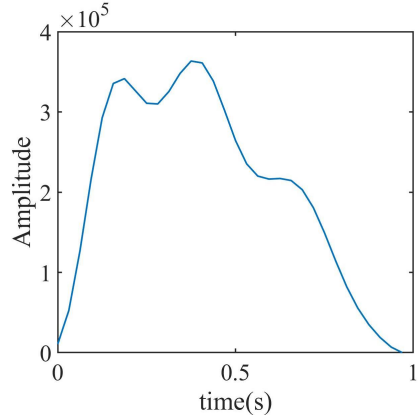

(b)
Fig. 5. Single period sample after executing FSW algorithm. (a) Single period valid sample, (b) Single period invalid sample.

\section{Feature Extraction based on Gaussian Fitting}

Waveform fitting has become a promising approach on contour analysis. It breaks down different waveforms into several different sub-waves [21], [22]. Gaussian function has been used to fit ear and finger PPG pulses [23]. Both researches demonstrate the rationality and effectiveness of fitting for analyzing waveform profiles. However, these studies were more concerned with the peak timing information of the curve and the calculation of the cardiovascular index based on the sub-waves. The physiological relevance of other characteristic features, including the half-width and the peak amplitude of the modeled Gaussian curves, is very less investigated. Therefore, we propose in this study to fit the PPG signals by Gaussian function to determine the characteristic parameters from the decomposed sub-waves, and then compare the morphological differences between PPG signals at the three blood glucose levels by statistical methods, inferring the correlationship between PPG signals and blood glucose. We categorize the single-period samples obtained in section III.C according to the blood glucose level divisions in Table I, and randomly select 200 samples in each group for subsequent analysis. Each PPG sample is then normalized in both amplitude (0-1) and width (50 sampling points).

Two positive Guassian functions $\left(f_{1}^{*}(n)\right.$ and $\left.f_{2}^{*}(n)\right)$ are applied to each normalized PPG sample $f(n)(n=1,2,3, \ldots, 50)$. They are defined as follows:

$$
f_{k}^{*}(n)=H_{k} \times \exp \left(-\frac{2\left(n-n_{k}\right)^{2}}{W_{k}^{2}}\right)
$$

where $H_{k}$ denotes the peak amplitude, $n_{k}$ represents the peak position and $W_{k}$ denotes the half-width of each Gaussian curve. $k=1,2$.

Fig. 6 demonstrates the Gaussian fitting for PPG waveforms at different blood glucose levels from normal (A1 and A2), borderline (B1 and B2) and warning (C1 and $\mathrm{C} 2)$. Six characteristic parameters from the two modeled Gaussian functions are also marked.

The least squares error (LSE) is used to assess the Gaussian fitting which is calculated by

$$
L S E=\sum_{n=1}^{30} \frac{\left[f_{1}^{*}(n)+f_{2}^{*}(n)-f(n)\right]^{2}}{f^{2}(n)} \times 100 \%
$$

Therefore it is evident that the smaller the fitting errors, the smaller are the parameter errors. The parameter optimization will not be stopped until the LSE is smaller than $1.5 \%$, which is accurate enough for waveform matching.

Table IV presents the comparison of six Gaussian features at different blood glucose levels. The peak amplitude and peak position of the first Gaussian curve have significant differences (both $P<0.05$ ), while the change in half-width is not obvious $(P=0.3)$. At the same time, as the blood glucose level increases, the peak amplitude appears a downward trend while the peak position moves forward. The PPG waveform is traditionally considered to contain both forward and backward components [24], [25]. The forward component is derived from the ejection of the left ventricle and is more likely to 

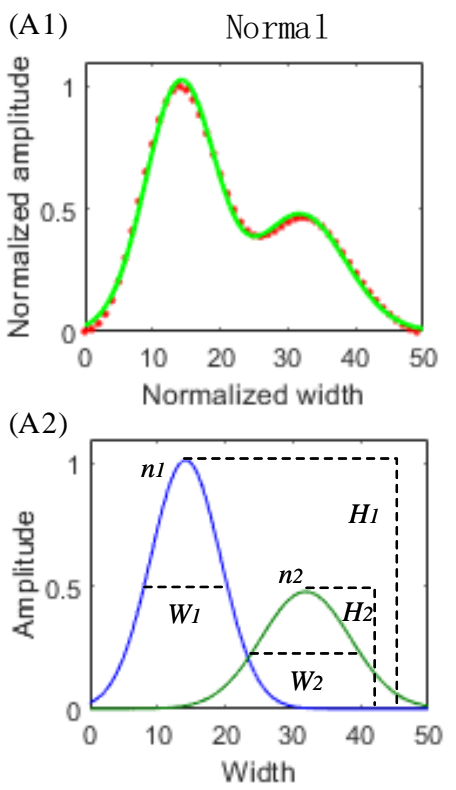
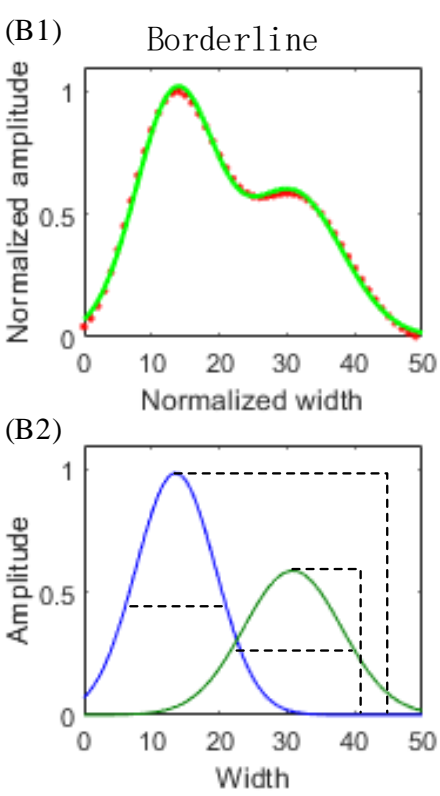

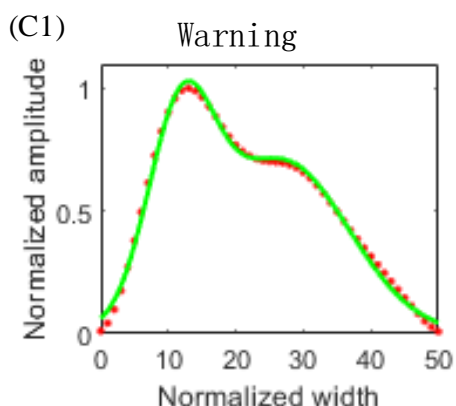

(C2)

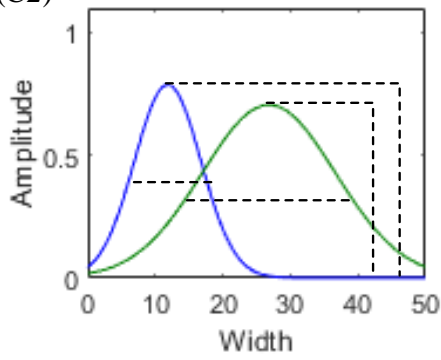

Fig. 6. Demonstration of Gaussian fitting for PPG waveforms: the normalized single period PPG signal $f(n)$ (red scatter) and the Gaussian fitting result (solid green line) from normal (A1), borderline (B1), and warning (C1) are shown in the upper panels. The two corresponding Gaussian functions $f_{1}^{*}(n)$ and $f_{2}^{*}(n)$ are shown in the bottom panels. The Gaussian features: peak amplitude: $H_{1}, H_{2}$, peak position: $n_{1}, n_{2}$ and half-width: $W_{1}, W_{2}$ are also shown.

TABLE IV

Comparison of Six Gaussian Features at Different Blood Glucose Levels.

\begin{tabular}{|c|c|c|c|c|c|c|}
\hline Variables & & normal & Blood & $\begin{array}{r}\text { Glucose } \\
\text { borderline }\end{array}$ & warning & P-Value \\
\hline \multirow[t]{2}{*}{ peak amplitude } & $H_{1}$ & $0.93 \pm 0.05$ & & $0.87 \pm 0.11$ & $0.76 \pm 0.10$ & $<0.05$ \\
\hline & $H_{2}$ & $0.47 \pm 0.13$ & & $0.61 \pm 0.07$ & $0.69 \pm 0.14$ & $<0.05$ \\
\hline \multirow[t]{2}{*}{ peak position } & $n_{1}$ & $15.4 \pm 2.1$ & & $13.7 \pm 1.5$ & $11.6 \pm 2.7$ & $<0.05$ \\
\hline & $n_{2}$ & $33.1 \pm 2.7$ & & $31.5 \pm 1.4$ & $28.2 \pm 2.6$ & $<0.05$ \\
\hline \multirow[t]{2}{*}{ half-width } & $W_{1}$ & $14.7 \pm 1.5$ & & $15.4 \pm 3.2$ & $15.8 \pm 2.6$ & 0.3 \\
\hline & $W_{2}$ & $15.1 \pm 1.6$ & & $17.5 \pm 2.1$ & $22.5 \pm 3.3$ & $<0.05$ \\
\hline
\end{tabular}

Note: Data are presented as mean \pm standard deviation (SD). $P$-value obtained from ANOVA analysis measures the differences between the three groups categorized by blood glucose. Statistical significance is set a priori at $\mathrm{P}<0.05$.

be related to the first Gaussian curve. In hyperglycemia, the blood is more viscous, the peripheral resistance of the heart is higher and the peak amplitude is slightly lower than that of normal people. We also observe that the peak amplitude, peak position and half-width of the second Gaussian curve are significantly different (all $P<0.05$ ). At the same time, as the blood glucose level increases, the peak amplitude gradually increases, the peak position moves forward and the half-width presents an increasing trend. The backward component may be related to the second Gaussian function, which may be caused by blood circulation from the peripheral circulation system. Due to the increase of blood flow resistance, the waveform will slow down in the process of blood flow to the heart, the waveform position is relatively high and the descending branch is gentle.

Based on the comparison of the six Gaussian characteristic parameters mentioned above, we extracted a total of 28 features in the time and frequency domains. Detailed feature definitions are shown in Table V. $H_{1}, H_{2}, n_{1}, n_{2}, W_{1}$, and $W_{2}$ are considered as features because they can visually show the difference among the three groups of data. In addition, other feature points can be determined by two Gaussian curves. For example, highest_peak represents the maximum amplitude of the signal, actually the peak position where the first Gaussian curve appears, determined by $n_{1}$. By Gaussian fitting, we conclude that different blood glucose levels will affect the slope and amplitude of the ascending and descending branches. Therefore, highest_peak, dis_peak and notch are used to represent the height characteristics of the waveform feature points. $n_{1}, n_{2}$, time_notch, timediff_peak_notch, timediff_notch_diastolicpeak and timediff_diastolicpeak_end together indicate changes in blood flow rate due to blood viscosity. The slop_rise, slop_fall, and slop_peak_diastolic are used to describe the slope of ascending and descending branches. area_single, area_start_max, area_max_notch, area_notch_diastolicpeak and area_diastolicpeak $k$ end reflect the difference in amplitude and time more intuitively. The width_period is an important feature for similar periodic signals, especially in distinguishing between valid signals and invalid signals. 
TABLE V

DEFINITIONS OF FEATURES.

\begin{tabular}{|c|c|}
\hline Feature & Definition \\
\hline$H_{1}$ & The peak of the first Gaussian curve \\
\hline $\mathrm{H}_{2}$ & The peak of the second Gaussian curve \\
\hline$n_{1}$ & The value of time when the amplitude is maximum \\
\hline$n_{2}$ & The value of time when diastolic occurs \\
\hline$W_{1}$ & The half-width of the first Gaussian curve \\
\hline$W_{2}$ & The half-width of the second Gaussian curve \\
\hline highest_peak & Maximum amplitude of the signal \\
\hline dis_peak & The amplitude of diastolic peak \\
\hline notch & The amplitude of the notch \\
\hline time_notch & The value of time when notch occurs \\
\hline width_period & Time taken for one period \\
\hline slop_rise & Rising rate of the single period from start to the peak \\
\hline slop_fall & Falling rate of the single period from peak to the end \\
\hline $\begin{array}{l}\text { slop_peak_dias- } \\
\text { tolic }\end{array}$ & Falling rate from peak to the diastolic peak \\
\hline $\begin{array}{l}\text { timediff_peak_- } \\
\text { notch }\end{array}$ & Total time it takes from peak to the notch \\
\hline $\begin{array}{l}\text { timediff_notch_- } \\
\text { diastolicpeak }\end{array}$ & Total time it takes from notch to the diastolic peak \\
\hline $\begin{array}{l}\text { timediff_dias- } \\
\text { tolicpeak_end }\end{array}$ & Total time it takes from diastolic peak to the end \\
\hline area_single & Area of the single period \\
\hline area_start_max & Area from start to the peak \\
\hline area_max_notch & Area from peak to the notch \\
\hline $\begin{array}{l}\text { area_notch_dias- } \\
\text { tolicpeak }\end{array}$ & Area from notch to the diastolic peak \\
\hline $\begin{array}{l}\text { area_diastolic- } \\
\text { peak end }\end{array}$ & Area from diastolic peak to the end \\
\hline
\end{tabular}

\section{EXPERIMENT}

\section{A. Dataset}

The research protocol was approved by Medical Ethics Committee of Qilu Hospital of Shandong University with approval code 2019-083. The dataset was collected from 80 subjects (37.5\% normal, $62.5 \%$ hyperglycemic) who were informed about the data collection procedure and signed an informed consent form. All patients have a history of more than one year without complications and confirm that they have not participated in any other 'clinical trial' within the previous three months. Subjects were asked to sit for three minutes to adjust their breathing and sitting position in order to reduce the impact of noise, and researchers recorded their height and weight measurements, as well as their age and medical history. The physical characteristics of the participants are listed in Table VI. Since the PPG signal is very sensitive to temperature and ambient light, data collection was undertaken in a quiet, temperature-controlled $\left(24 \pm 2^{\circ} \mathrm{C}\right)$ and no strong light laboratory. All the subjects were uniquely numbered based on the measured blood glucose values, with individual numbers 1-30 in the G1 group, 31-58 in the G2 group, and 59-80 in the G3 group.

\section{B. Analysis and Diagnosis}

In our experiment, single-period samples acquired from FSW algorithm contain both valid samples and invalid ones. In order to improve the blood glucose classification accuracy, invalid samples should be identified and eliminated by binary classification. The Gaussian Support Vector Machine (GSVM),
TABLE VI

PARTICIPANTS PHYSICAL CHARACTERISTICS.

\begin{tabular}{lllll}
\hline Measurement & Max & Min & Mean & Std.Deviation \\
\hline Age(years) & 65 & 37 & 47.61 & 5.79 \\
Weight $(\mathrm{kg})$ & 86.7 & 58.5 & 64.87 & 10.58 \\
Height $(\mathrm{m})$ & 1.9 & 1.55 & 1.712 & 0.14 \\
BMI $(\mathrm{kg} / \mathrm{m} 2)$ & 27.36 & 17.58 & 23.56 & 4.45 \\
Glucose $(\mathrm{mg} / \mathrm{dl})$ & 11 & 3.9 & 7.86 & 2.16 \\
\hline
\end{tabular}

Bagged Trees (BT) and K-nearest neighbor (KNN) classifiers were trained with 122 invalid samples and 140 valid samples from individuals numbered 1-15, 31-44, and 59-69. The above classifiers were tested with 114 invalid samples and 130 valid samples from the remaining individuals numbered 16-30, 4558 , and 70-80. The testing accuracy of the three classifiers was $97.54 \%, 96.31 \%$ and $90.57 \%$, respectively, as shown in Table VII. A total of 6264 samples from 80 subjects were fed as input of the GSVM model. 476 samples identified as invalid ones were subsequently removed, and the remaining 5788 valid samples were adopted for subsequent analysis.

2,930 samples (from individuals 1-15, 31-44, 59-69) were used to train GSVM, BT and KNN classifiers. The labels of the training samples were set to be $-1,0$ and 1 , representing the G1, G2 and G3 blood glucose groups, respectively. Fivefold cross-validation was used in the experiment to reduce the impact of sample partitioning. The implementation result is listed in table VIII. The test accuracy of 2858 samples from the remaining 40 individuals are $81.49 \%, 74 \%$ and $71.27 \%$, respectively. It indicates that GSVM has the best effect for PPG signal classification.

In the subsequent analysis, we used a GSVM-based classification model as shown in Eq. (5). The value $C$ is the predicted class of GSVM. The coding matrix $M$ represents the coding relationship between each binary SVM model $f(x)=w^{T} x+b$ and various classes of signals in the corresponding data set. Its matrix element is $m_{k l}$, where $l$ represents a binary SVM and $k$ represents a data set category. $g$ is the loss of decoding scheme. $m_{l}$ is the predicted score of the SVM $l$ whose prediction result is chosen according to $m_{k l}$ [26].

$$
\begin{gathered}
C=\operatorname{argmin} \frac{\sum_{l=1}^{L}\left|m_{k l}\right| g\left(m_{k l}, s_{l}\right)}{\sum_{l=1}^{L}\left|m_{k}\right|} \\
M=\left[\begin{array}{ccc}
1 & -1 & 0 \\
1 & 0 & -1 \\
0 & 1 & -1
\end{array}\right]
\end{gathered}
$$

The Gaussian kernel function of the SVM is presented in Eq. (6), which maps finite-dimensional data to highdimensional space. The $\left\|x-x^{\prime}\right\|$ is the square Euclidean distance between two vectors. $\sigma$ is a free parameter that controls the radial range of action, where our $\sigma$ is set to 0.05 .

$$
\begin{gathered}
k\left(x, x^{\prime}\right)=e^{-\frac{\left\|x-x^{\prime}\right\|^{2}}{2 \sigma^{2}}} \\
P(i)=\left\{\begin{array}{cc}
-1 & n_{1, i}>n_{2, i}, n_{3, i} \\
0 & n_{2, i}>n_{1, i}, n_{3, i} \\
1 & n_{3, i}>n_{1, i}, n_{2, i}
\end{array}\right.
\end{gathered}
$$


TABLE VII

The Results of InVAlid SAMPle Classification.

\begin{tabular}{llllll}
\hline Classifier & Precision & Sensitivity & Specificity & F $_{1}$ Score & Accuracy \\
\hline GSVM & $96.97 \%$ & $98.46 \%$ & $96.49 \%$ & $97.71 \%$ & $97.54 \%$ \\
BT & $95.49 \%$ & $97.69 \%$ & $94.74 \%$ & $96.58 \%$ & $96.31 \%$ \\
KNN & $89.05 \%$ & $93.85 \%$ & $86.84 \%$ & $91.39 \%$ & $90.57 \%$ \\
\hline
\end{tabular}

TABLE VIII

The Results of Blood Glucose Level Classification.

\begin{tabular}{llllll}
\hline Classifier & Precision & Sensitivity & Specificity & F 1 Score & Accuracy \\
\hline GSVM & $80.84 \%$ & $79.58 \%$ & $83.19 \%$ & $80.2 \%$ & $81.49 \%$ \\
BT & $73.05 \%$ & $71.05 \%$ & $76.64 \%$ & $72.03 \%$ & $74 \%$ \\
KNN & $69.23 \%$ & $70.3 \%$ & $72.14 \%$ & $69.76 \%$ & $71.27 \%$ \\
\hline
\end{tabular}

In the actual clinical diagnosis, the value of the diagnosis result $P(i)$ is $-1,0$ or 1 , which indicates that the blood glucose level is normal, borderline and warning respectively. In Eq. (7), $n_{1, i}, n_{2, i}$ and $n_{3, i}$ represent the normal, borderline and warning blood glucose level samples of user $i$. When the number of samples of a certain blood glucose level is greater than the number of samples of the other two types, the user is judged to be at the blood glucose level.

Testing was implemented by those subjects numbered 1630, 45-58 and 70-80, who did not participate in the classification model training. Each subject recorded a 60 -second video by our smartphone program, which was then automatically uploaded to the cloud (PC workstation with 3.70-GHz CPU, 1080Ti GPU and 64-GB RAM) via wireless communication for PPG signal extraction, signal processing and classification. The classification results, as summarized in Table IX, were returned to the user smartphone. All single-period samples from one subject were fed into the trained GSVM model. Using the extracted features, 11 of the 15 normal subjects were correctly classified, and 3 of them were mistakenly put into the borderline group. Among the 14 subjects with borderline blood glucose level, 10 were correctly identified. As for the 10 subjects with warning blood glucose level, 9 were correctly classified, offering an accuracy rate of $90 \%$. This result validates the effectiveness of the features extracted and is crucial for identifying blood glucose level of the warning status. For the purpose of prompt response, we only collected 60 -second video, accordingly the average number of effective samples per individual is 64 . On calculating the correct probability of classification, the possibility of misdiagnosis will increase due to the small sample size. In order to provide users with a more reliable reference, three consecutive measurements are recommended. If three consecutive measurements fall into the same category, then we can reasonably believe the evaluation results. Once the three evaluation results are not the same, however, then the user is recommended to perform an invasive blood glucose meter test.

\section{CONCLUSION}

This work introduces a non-invasive blood glucose monitoring system based on smartphone PPG signals and machine
TABLE IX

The Prediction Results of GSVM.

\begin{tabular}{|c|c|c|c|c|}
\hline \multicolumn{2}{|c|}{ GSVM } & \multicolumn{3}{|c|}{ True level } \\
\hline & & normal & borderline & warning \\
\hline & normal & 11 & 1 & 0 \\
\hline Prediction & borderline & 3 & 10 & 1 \\
\hline level & warning & 1 & 3 & 9 \\
\hline \multicolumn{2}{|c|}{ Accuracy } & $73.33 \%$ & $71.43 \%$ & $90.00 \%$ \\
\hline
\end{tabular}

learning algorithms. It builds up a good basis for real-time monitoring of human physiological parameters in the home environment. Firstly, a left-hand index finger video is recorded using a smartphone and PPG signals are extracted from the video. After that the FSW algorithm removes baseline drift and divides the pre-processed signals into single periods. The result of the segmented PPG signals demonstrates the effectiveness of the developed FSW algorithm. Secondly, Gaussian fitting method is used to extract 28 features of each single-period signal in time domain and frequency domain. Finally, the blood glucose is classified as normal, borderline or warning level by machine learning classifier with the accuracy of $81.49 \%$, which means that the 28 features extracted are effective in separating samples of different blood glucose levels.

One of our future works is to divide the blood glucose range into more intervals to offer more precise feedbacks to the users. Therefore the PPG signal data will be augmented using Generative Adversarial Networks (GAN) to satisfy the input requirement of deep learning networks. We also wish to find more feasible features for more exact blood glucose detection. For instance, according to the hemodynamics theory, there will be energy changes in the blood during the flow, which is particularly prominent at different blood glucose levels. Therefore, Kaiser-Teager Energy feature, Spectral Entropy feature and Spectral Energy Logarithmic feature are under consideration for possible involvement. In addition, we will consider the correlationship between the features to eliminate redundant features especially for real time response. 


\section{REFERENCES}

[1] S. Ramasahayam, L. Arora, S. R. Chowdhury, and M. Anumukonda, "Fpga based system for blood glucose sensing using photoplethysmography and online motion artifact correction using adaline," 2015 9th International Conference on Sensing Technology (ICST), pp. 22-27, Dec 2015.

[2] S. A. Siddiqui, Y. Zhang, J. Lloret, H. Song, and Z. Obradovic, "Painfree blood glucose monitoring using wearable sensors: Recent advancements and future prospects," IEEE Reviews in Biomedical Engineering, vol. 11 , pp. 21-35, 2018.

[3] S. Sinchai, P. Kainan, P. Wardkein, and J. Koseeyaporn, "A photoplethysmographic signal isolated from an additive motion artifact by frequency translation," IEEE Transactions on Biomedical Circuits and Systems, vol. 12, no. 4, pp. 904-917, Aug 2018.

[4] P. P. Pai, P. K. Sanki, S. K. Sahoo, A. De, S. Bhattacharya, and S. Banerjee, "Cloud computing-based non-invasive glucose monitoring for diabetic care," IEEE Transactions on Circuits and Systems I: Regular Papers, vol. 65, no. 2, pp. 663-676, Feb 2018.

[5] S. Lekha and S. M, "Real-time non-invasive detection and classification of diabetes using modified convolution neural network," IEEE Journal of Biomedical and Health Informatics, vol. 22, no. 5, pp. 1630-1636, Sep. 2018.

[6] J. Harvey, S. M. A. Salehizadeh, Y. Mendelson, and K. H. Chon, "Oxima: A frequency-domain approach to address motion artifacts in photoplethysmograms for improved estimation of arterial oxygen saturation and pulse rate," IEEE Transactions on Biomedical Engineering, vol. 66, no. 2, pp. 311-318, Feb 2019.

[7] S. A. Siddiqui, Y. Zhang, Z. Feng, and A. Kos, "A pulse rate estimation algorithm using ppg and smartphone camera," Journal of Medical Systems., vol. 40, no. 5, pp. 1-6, May 2016.

[8] K. T. Tanweer, S. R. Hasan, and A. M. Kamboh, "Motion artifact reduction from ppg signals during intense exercise using filtered x-lms," 2017 IEEE International Symposium on Circuits and Systems (ISCAS), pp. 1-4, May 2017.

[9] P. M. Nabeel, S. Karthik, J. Joseph, and M. Sivaprakasam, "Arterial blood pressure estimation from local pulse wave velocity using dualelement photoplethysmograph probe," IEEE Transactions on Instrumentation and Measurement, vol. 67, no. 6, pp. 1399-1408, June 2018.

[10] M. A. Motin, C. K. Karmakar, and M. Palaniswami, "Ensemble empirical mode decomposition with principal component analysis: A novel approach for extracting respiratory rate and heart rate from photoplethysmographic signal," IEEE Journal of Biomedical and Health Informatics, vol. 22, no. 3, pp. 766-774, May 2018.

[11] A. Hernando, M. D. Peláez, M. T. Lozano, M. Aiger, E. Gil, and J. Lázaro, "Finger and forehead ppg signal comparison for respiratory rate estimation based on pulse amplitude variability," 2017 25th European Signal Processing Conference (EUSIPCO), pp. 2076-2080, Aug 2017.

[12] A. Hina, H. Nadeem, and W. Saadeh, "A single led photoplethysmography-based noninvasive glucose monitoring prototype system," 2019 IEEE International Symposium on Circuits and Systems (ISCAS), pp. 1-5, May 2019.

[13] E. Monte-Moreno, "Non-invasive estimate of blood glucose and blood pressure from a photoplethysmograph by means of machine learning techniques," Artificial Intelligence in Medicine, vol. 53, no. 2, pp. 127 $-138,2011$.

[14] C. Tsai, C. Li, R. W. Lam, C. Li, and S. Ho, "Diabetes care in motion: Blood glucose estimation using wearable devices," IEEE Consumer Electronics Magazine, vol. 9, no. 1, pp. 30-34, Jan 2020.

[15] V. P. Rachim and W.-Y. Chung, "Wearable-band type visible-near infrared optical biosensor for non-invasive blood glucose monitoring," Sensors and Actuators B: Chemical, vol. 286, pp. 173 - 180, 2019.

[16] F. Lamonaca, Y. Kurylyak, D. Grimaldi, and V. Spagnuolo, "Reliable pulse rate evaluation by smartphone," 2012 IEEE International Symposium on Medical Measurements and Applications Proceedings, pp. 1-4, May 2012.

[17] J. Dey, A. Gaurav, and V. N. Tiwari, "Instabp: Cuff-less blood pressure monitoring on smartphone using single ppg sensor," 2018 40th Annual International Conference of the IEEE Engineering in Medicine and Biology Society (EMBC), pp. 5002-5005, July 2018.

[18] F. Tabei, R. Kumar, T. N. Phan, D. D. McManus, and J. W. Chong, "A novel personalized motion and noise artifact (mna) detection method for smartphone photoplethysmograph (ppg) signals," IEEE Access, vol. 6, pp. 60498-60512, 2018.
[19] A. Sološenko, A. Petrẻnas, and V. Marozas, "Photoplethysmographybased method for automatic detection of premature ventricular contractions," IEEE Transactions on Biomedical Circuits and Systems, vol. 9, no. 5, pp. 662-669, Oct 2015.

[20] H. Wang, X. Wang, J. R. Deller, and J. Fu, "Shape-preserving preprocessing for human pulse signals based on adaptive parameter determination," IEEE Transactions on Biomedical Circuits and Systems, vol. 8, no. 4, pp. 594-604, Aug 2014.

[21] S. C. Millasseau, J. M. Ritter, K. Takazawa, and P. J. Chowienczyk, "Contour analysis of the photoplethysmographic pulse measured at the finger," IEEE Transactions on Instrumentation and Measurement, vol. 24, pp. 1449-1456, Aug. 2006.

[22] D. Jang, S. Park, and M. Hahn, "A gaussian model-based probabilistic approach for pulse transit time estimation," IEEE Journal of Biomedical and Health Informatics, vol. 20, no. 1, pp. 128-134, Jan 2016.

[23] U. Rubins, "Finger and ear photoplethysmogram waveform analysis by fitting with gaussians," Medical \& Biological Engineering \& Computing, vol. 46, no. 12, pp. 1271-1276, Dec 2008.

[24] K. Soerensen, A. K. Verma, A. Blaber, J. Zanetti, S. E. Schmidt, J. J. Struijk, and K. Tavakolian, "Challenges in using seismocardiography for blood pressure monitoring," 2017 IEEE Computing in Cardiology (CinC), pp. 1-4, Sep. 2017.

[25] H. Wu, C. Lee, A. Liu, W. Chung, C. Tang, C. Sun, and H. Yip, "Arterial stiffness using radial arterial waveforms measured at the wrist as an indicator of diabetic control in the elderly," IEEE Transactions on Biomedical Engineering, vol. 58, no. 2, pp. 243-252, Feb 2011.

[26] I. Steinwart, D. Hush, and C. Scovel, "An explicit description of the reproducing kernel hilbert spaces of gaussian rbf kernels," IEEE Transactions on Information Theory, vol. 52, no. 10, pp. 4635-4643, Oct 2006. 